VII Congresso Brasileiro de Informática na Educação (CBIE 2018)

Anais do XXIV Workshop de Informática na Escola (WIE 2018)

\title{
Uso da Khan Academy como Avaliação Continuada em Cálculo I
}

\author{
Antonio Igor Oliveira, Elvis Medeiros de Melo, Josenaldo Silva Junior
}

Instituto Metrópole Digital - Universidade Federal do Rio Grande do Norte (UFRN) Av. Sen. Salgado Filho, 3000 - Lagoa Nova, CEP: 59.078-970 - Natal - RN - Brasil

igoroliveira@imd.ufrn.br, elvismedeiros.mm@gmail.com, josenaldo@ufrn.edu.br

\begin{abstract}
The discipline of Calculus I of the Bachelor of Information Technology (BTI) usually presents high failure rates. Based on this scenario, the viability and the necessity of the implementation of auxiliary methodologies of approach of the discipline become evident. Thus, the use of the Khan Academy platform, the main focus of this article, and Monitoring Calculus I has as main objective contributing to the improvement of the students' learning process and, consequently, decreasing the failure rates in the discipline. The methodology used is the use of online activities of the platform as a continuous evaluation throughout the semester. Satisfactory results were obtained, such as increasing the level of approval after application of the method.

Resumo. A disciplina de Cálculo I do Bacharelado em Tecnologia da Informação (BTI) costuma apresentar taxas de reprovação elevadas. Com base nesse cenário, a viabilidade e a necessidade da implantação de metodologias auxiliares de abordagem da disciplina tornam-se evidentes. Assim, o uso da plataforma Khan Academy, foco principal deste artigo, e da Monitoria de Cálculo I tem como principal objetivo contribuir para a melhora do processo de aprendizagem dos alunos $e$, consequentemente, diminuir as taxas de reprovação na disciplina. A metodologia empregada consiste no uso de atividades online da plataforma como uma avaliação continuada ao longo do semestre. Obtiveram-se resultados satisfatórios, como o aumento do nível de aprovação após aplicação do método.
\end{abstract}

\section{Introdução}

O avanço da tecnologia propicia inúmeras possibilidades de desenvolvimento para a sociedade. Além de viabilizar a automação de processos e aumentar significativamente a produtividade de vários segmentos da economia, o avanço tecnológico provoca, ainda, mudanças radicais na forma como se dá a interação entre as pessoas. Uma dessas mudanças, tendo em vista o contexto educacional e o advento da tecnologia e avanços da Web 2.0, é o e-learning, uma modalidade de ensino a distância por meio de ferramentas digitais. Atualmente, diversas ferramentas com essa perspectiva estão sendo desenvolvidas, entre elas a Khan Academy (KA) ${ }^{1}$.

A Khan Academy é uma plataforma online que disponibiliza módulos de estudos, entre eles vídeos com conteúdos sobre Álgebra, Aritmética, Geometria, Biologia, Física, Cálculo I, etc. A forma em que a Khan Academy disponibiliza o conhecimento para as pessoas se baseia em exercícios práticos, que variam de acordo com a matéria selecionada, e videoaulas. A iniciativa da KA, além de chamar atenção pelo sucesso, traz reflexões acerca dos modelos educacionais tradicionais. Os modelos nos quais o professor é o principal responsável pela transmissão do conhecimento e os alunos, passivos no processo de aprendizagem não se

${ }^{1}$ Disponível em: 〈https://pt.khanacademy.org/〉. Acesso em: 29 jun. 2018 
VII Congresso Brasileiro de Informática na Educação (CBIE 2018)

Anais do XXIV Workshop de Informática na Escola (WIE 2018)

sustentam mais [Morin 2015], e são influenciados pelas Tecnologias Digitais de Informação e Comunicação (TDICs). Uma metodologia adotada por plataformas similares à KA é a elearning.

A metodologia e-learning adotada pela Khan Academy tem potencial para o ensino de disciplinas da área tecnológica que geralmente possuem dificuldade de aprendizagem, segundo Medeiros Filho e Moura (2016). A Matemática se encaixa perfeitamente nesse quadro, principalmente a que é lecionada em disciplinas de Cálculo e em outros componentes curriculares no ensino superior.

Em se falando de aprendizagem matemática em um contexto regional, segundo dados da Plataforma QEdu, que se baseia em avaliações de larga escala aplicadas aos estudantes brasileiros de escolas públicas, apenas $20 \%$ dos alunos potiguares do $5^{\circ}$ ano do Ensino Fundamental possuem aprendizado matemático adequado. Esse índice é ainda pior para o $9^{\circ}$ ano, em que somente $8 \%$ dos discentes têm a proficiência matemática indicada para a faixa etária escolar [QEDU 2018]. Isso implica que a quase totalidade dos estudantes do estado chega à última fase do Ensino Fundamental com sérias dificuldades em Matemática, cujas consequências evidenciam-se nos ensinos Médio e Superior. Em relação ao segundo, as disciplinas de base, como Cálculo I, não são exitosas ao suprir a carga teórica não aprendida nas fases anteriores da vida escolar do aluno.

A disciplina de Cálculo I do Bacharelado em Tecnologia da Informação (BTI) costuma apresentar taxas de reprovação elevadas. Com base nesse cenário, a viabilidade e a necessidade da implantação de metodologias auxiliares de abordagem da disciplina tornam-se evidentes. Estratégias de e-learning que adotam a plataforma Khan Academy vêm sendo disseminadas no contexto brasileiro, dando ênfase ao surgimento de práticas educativas no ensino superior em disciplinas tecnológicas. Um exemplo é proposto por Menegais, Fagundes e Sauer (2015), no qual utilizaram a plataforma para promover a formação continuada de professores de Matemática para o uso das TDICs no âmbito de suas salas de aula, e, também, para a sua própria atualização. O uso da plataforma proporcionou uma melhoria no processo de ensino dos professores e de aprendizagem dos alunos, segundo dados coletados. Bardy et al (2007) defendem que o professor deve assumir o papel de estimulador, mediador que coordena as discussões das ideias que vão sendo construídas, instigando os alunos a fazerem novas descobertas a partir da interação com colegas e com as TDICs.

A Plataforma KA oferece a possibilidade de o professor criar uma turma virtual, bem como acompanhar em tempo real o desempenho dos estudantes, por meio do software disponibilizado pela plataforma, recurso esse que difere a KA de outras plataformas de elearning. Assim, o uso da plataforma Khan Academy (o foco principal deste artigo) e da Monitoria de Cálculo I tem como principal objetivo contribuir para a melhora do processo de aprendizagem dos alunos e, consequentemente, diminuir as taxas de reprovação na disciplina. Apresentaremos, no decorrer do artigo, o impacto na taxa de aprovação nos três semestres nos quais se aplicou o método e alguns resultados detalhados para duas turmas de Cálculo I do BTI no segundo semestre de 2017.

O presente artigo está estruturado da seguinte forma: esta introdução; fundamentação teórica acerca da proposta do trabalho; metodologia desenvolvida; resultados e discussões, seção na qual discorremos acerca dos resultados; considerações sobre a prática; e referências consultadas. 
VII Congresso Brasileiro de Informática na Educação (CBIE 2018)

Anais do XXIV Workshop de Informática na Escola (WIE 2018)

\section{Fundamentação Teórica}

Quando direcionamos o olhar para o ensino da Matemática, identificamos problemas alarmantes. Segundo relatório publicado pela Organização para a Cooperação e Desenvolvimento Econômico (OCDE), o Brasil está entre os dez países com pior rendimento escolar em Matemática, Ciências e Leitura [OCDE 2016]. Ainda segundo o estudo, 67,1\% dos alunos brasileiros apresentam rendimento e proficiência em Matemática baixos. O uso de metodologia e-learning tem potencial para reverter esse quadro. Tudo vai depender da postura do professor frente a essas tecnologias e como vai mediá-las no processo de ensino [Bardy et al 2007].

A problemática evidenciada pelo relatório do OCDE reflete diretamente no desempenho dos alunos no Ensino Superior, e, portanto, não é de se surpreender que experiências envolvendo a disciplina de Cálculo I venham sendo disseminadas no âmbito da pesquisa acadêmica. Pavanelo e Lima (2017) trazem uma experiência de sala de aula invertida utilizando a ferramenta Khan Academy em Cálculo I. Elas apontaram potencialidades da ferramenta como introdução de conceitos relacionados a disciplina, e em sala de aula havia discussões acerca das situaçõesproblema vivenciadas durante o uso da ferramenta e-learning. Elas apontam, também, a necessidade de práticas inovadoras em relação ao ensino de Cálculo I com suporte de tecnologia no ensino superior.

Castro-Filho et al (2011, p.14) destacam que “[...] um conceito matemático pode influenciar o desenvolvimento de ferramentas tecnológicas, mas posteriormente pode ser influenciado por essa mesma tecnologia”. É possível propor uma articulação entre os métodos de e-learning e os processos de aprendizagem de conteúdos matemáticos. Para Morin (2015), muitas formas de se ensinar não se justificam mais, e é necessário repensar o modelo de aula tradicional enraizado nos processos de ensino, principalmente no que tange ao da Matemática.

A busca por métodos inovadores que contribuam para a superação de dificuldades encontradas no ensino de Cálculo I e que motivem e envolvam o estudante em seu processo de desenvolvimento dos raciocínios matemáticos é um desafio. Para Becker (2012), a construção de conceitos com suporte das TDICs é possível. Existe um aumento na oferta de recursos tecnológicos na sala de aula, que, muitas vezes, não são integrados efetivamente às práticas pedagógicas dos professores, muito menos ao processo de aprendizagem dos estudantes [Menegais, Fagundes e Sauer 2015]. A plataforma KA proporciona softwares que identificam o estilo de aprendizagem de cada aluno de acordo com os feedbacks dados à plataforma, além de sugerir exercícios coerentes aos conteúdos estudados.

Pavanelo e Lima (2017) relatam que, ao longo da história da implantação da disciplina de Cálculo I no Ensino Superior, a questão do nível de rigor simbólico-formal a ser adotado sempre foi um elemento central nas discussões sobre o processo de ensino e aprendizagem dessa disciplina. Segundo os autores, há dois cenários que merecem destaque: ou os alunos demonstram dificuldades para acompanhá-la, advindas do alto nível de rigor simbólico-formal, ou a metodologia utilizada não possibilita que os alunos desenvolvam todo o seu potencial em relação aos conteúdos da disciplina. Apesar das dificuldades em relação à metodologia com base na qual a disciplina é ministrada, há experiências exitosas na área, inclusive utilizando a ferramenta Khan Academy.

Ao aplicarem um questionário a fim de reconhecer o tempo semanal dedicado aos estudos de Cálculo I fora do ambiente da sala de aula, Pavanelo e Lima (2017) tiveram como resultados: $10 \%$ dos alunos disseram que estudavam mais de 8 horas semanais, $20 \%$ entre 4 e 8 
VII Congresso Brasileiro de Informática na Educação (CBIE 2018)

Anais do XXIV Workshop de Informática na Escola (WIE 2018)

horas, $50 \%$ entre 2 e 4 horas, e 20\% menos de 2 horas semanais. Esses dados foram influenciados, segundo os autores e os dados coletados em outras perguntas, pelo uso da Khan Academy. Os alunos que dedicaram mais tempo aos estudos extra-classe foram os que tiveram mais êxito na avaliação escrita.

Ao analisarem a proposta pedagógica como um todo da KA, Tavares et al (2012) consideraram diversas metodologias acerca de práticas pedagógicas, discorrendo sobre a importância do não engessamento de uma única prática no processo de ensino, para levar em consideração as diferentes formas de aprendizagem de um indivíduo possíveis. Além disso, eles consideram que a KA tem vários itens que a caracterizam com abordagem construtivista, embora tenha alguns aspectos alinhados à abordagem tradicional. Os autores terminam argumentando que ainda não há um consenso quanto aos procedimentos a serem adotados na construtivista, e que ambas as abordagens são complementares. Além disso, tudo vai depender do nível de abordagem e visão do professor ao planejar sua aula com o suporte da plataforma [Bardy et al 2007].

A seguir, apresentaremos a metodologia utilizada na pesquisa.

\section{Metodologia}

O professor aplicou atividades online logo após o término de cada conteúdo, e as atividades tinham prazo de entrega até a próxima aula presencial de cada turma. Essa prática se repetiu para todas as aulas do semestre e é o principal ponto da metodologia que apresentaremos aqui. A prática foi adotada com o intuito de potencializar o estudo continuado por parte dos alunos na disciplina de Cálculo I no segundo semestre de 2017.

O primeiro estímulo para os alunos foi transformar essas atividades em uma avaliação continuada que comporia parte da nota de cada unidade da disciplina. A Khan Academy foi fundamental pois, além de disponibilizar as atividades online, dispõe de ferramentas para que o professor acompanhe o desempenho dos seus alunos.

Por meio de um cadastro na KA, o professor criou uma turma virtual e disponibilizou o código para os alunos se cadastrarem. Feito isso, passou a obter vários tipos de informação sobre eles. Uma das informações mais relevantes é o progresso de cada aluno nas atividades. A plataforma informa quantas questões por atividade o estudante tentou, o tempo gasto em cada questão, quais ele acertou na primeira tentativa, em quais ele assistiu a um vídeo ou fez uso de dicas para ajudá-lo, qual foi a maior sequência de acertos e a porcentagem de acertos nos últimos dez problemas tentados. A Figura 1 ilustra como esses dados são mostrados na área do professor. Os retângulos azuis representam acertos no problema, e os vermelhos representam erros ou uso de dicas específicas para a solução do problema. Ademais, o ícone acima do retângulo do problema nove indica que o aluno assistiu a uma das videoaulas da KA sobre aquele assunto durante a resolução do problema. 
VII Congresso Brasileiro de Informática na Educação (CBIE 2018)

Anais do XXIV Workshop de Informática na Escola (WIE 2018)

Habilidades » Limites laterais a partir de gráficos
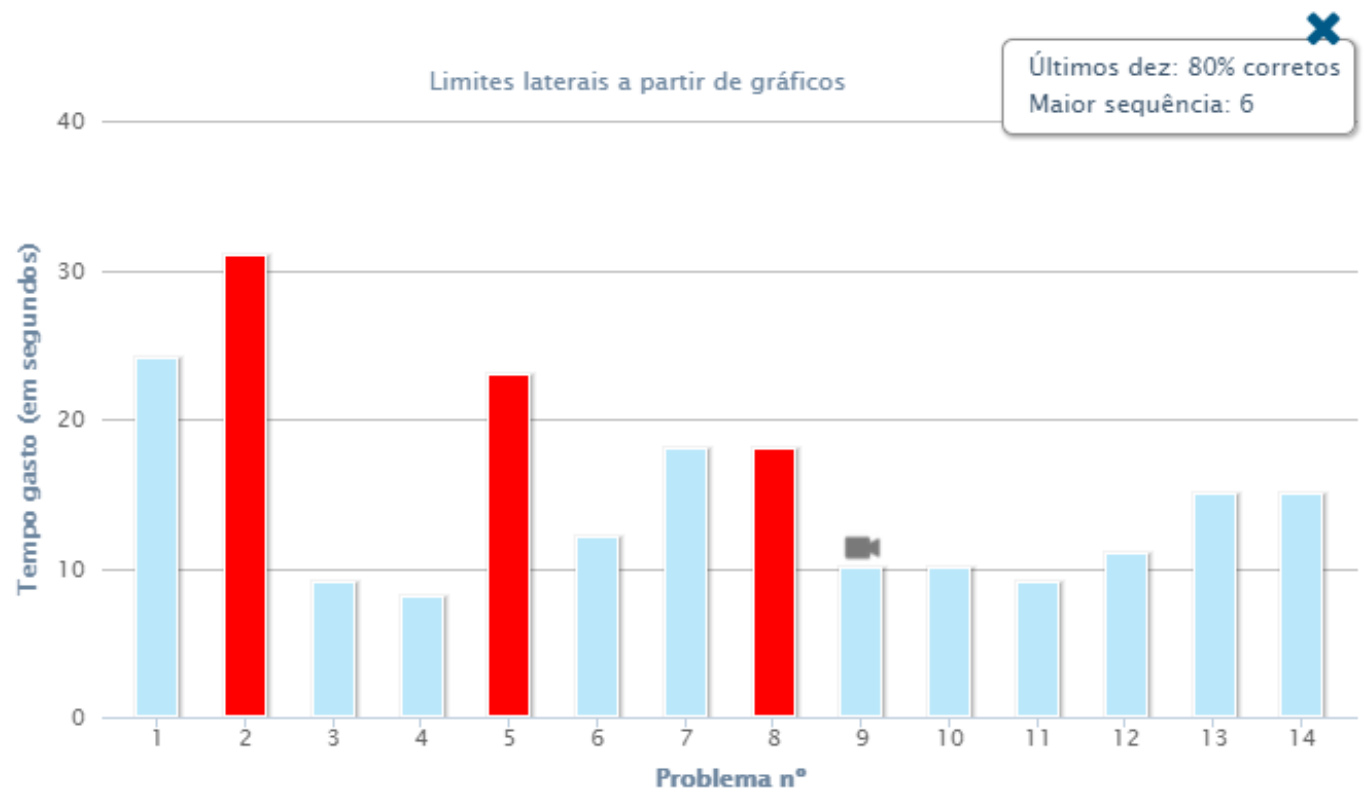

Figura 1. Relato de desempenho individual obtido na KA

Com os dados de acertos e erros nos problemas, foi criado um método avaliativo que atribuía uma nota de 0 (zero) a 100 pontos para cada atividade. Caso o aluno realizasse até cinco questões, recebia 20 pontos por cada acerto. No caso em que ele respondesse mais de cinco questões, a pontuação correspondia à porcentagem de acertos nos últimos dez problemas. Esse tipo de avaliação incentiva o aluno que comece mal uma atividade a recuperar sua nota e, consequentemente, melhorar seu aprendizado, conforme a abordagem construtivista proposta por Papert (2008). No cenário ilustrado na Figura 1, por exemplo, se o aluno parasse na quinta questão, seu desempenho seria de 60 pontos. Contudo, em virtude da persistência na atividade, ele teve a oportunidade de subir sua nota para 80 pontos. Além disso, se ele obtivesse mais um acerto no $15^{\circ}$ problema, subiria sua nota para 90 pontos, e alcançaria 100 pontos se acertasse do $15^{\circ}$ ao $18^{\circ}$.

Todas as pontuações foram registradas manualmente em uma planilha do Google, disponibilizada para os estudantes com o intuito de que eles acompanhassem suas notas. No final de cada unidade, uma pontuação final de 0 (zero) a 3,0 (três) pontos foi atribuída às atividades online proporcionalmente à média aritmética da pontuação de todas as atividades. Na primeira unidade, foram disponibilizadas 25 atividades, ao passo que, na segunda e na terceira, esse número foi de 15 e 13 , respectivamente.

Antes da aplicação dessa prática de ensino com a KA, as turmas de Cálculo I foram avaliadas com provas escritas totalizando 12,0 (doze) pontos, tendo, assim, dois pontos extras. No semestre 2016.2, quando se utilizou a KA pela primeira vez, a avaliação escrita passou a ter 10,0 pontos, e as atividades online, 3,0 pontos, como explicado anteriormente. Dessa forma, as avaliações passaram a ter 3,0 pontos extras. A priori, essa distribuição de notas foi pensada esperando uma adesão mediana às atividades da KA, dando ao estudante a oportunidade de obter a nota máxima somente com a prova escrita. No entanto, a adesão foi massiva, e apresentou-se o primeiro problema, que era o de como balancear a avaliação dos alunos. 
No segundo semestre de execução, a nota máxima das atividades online continuou a ser 3,0 pontos, mas a nota da avaliação escrita foi reduzida para 8,0 pontos, totalizando 11,0 pontos por unidade. Considerando a possibilidade de ser aprovado com média 5 em disciplinas da Universidade Federal do Rio Grande do Norte (UFRN), a avaliação foi julgada como tendo nível de dificuldade baixa. Na terceira e última execução dessa metodologia até o presente artigo, as pontuações foram mantidas, porém a avaliação escrita não foi composta classicamente por questões de nível fácil, médio e difícil. Foi avisado aos alunos que haveria somente questões médias e difíceis, indicando que a pontuação "fácil" seria atribuída às atividades online. Dessa forma, o método avaliativo apresentou outras falhas. As atividades online são, em sua totalidade, compostas por problemas objetivos nos quais se avaliam apenas a resposta final. Todo o desenvolvimento e fundamentação das soluções dos estudantes eram avaliadas nas provas escritas, mas a composição informada dificultou a avaliação desses aspectos em níveis mais baixos. No fim, essa forma de avaliação apresentou um nível de dificuldade adequado mas trouxe dificuldades na identificação de deficiências dos estudantes, com exceção daqueles que procuraram os atendimentos individuais com o docente ou a monitoria da disciplina.

\section{Resultados e Discussões}

A análise geral do impacto na taxa de aprovação trará a distinção de dois tipos de turmas. $\mathrm{O}$ motivo da distinção é o que os índices de abandono nas turmas compostas, em sua maioria, por alunos desnivelados no curso são maiores do que naquelas compostas por alunos nivelados, o que afeta suas taxas de aprovação. Logo, a separação dos tipos possibilita que sejam feitas comparações mais adequadas. Referir-nos-emos às turmas como "desniveladas" e "niveladas".

As taxas de aprovações apresentadas no Gráfico 1 mostram que, a partir da primeira execução, em 2016.2, da prática de ensino utilizando a Khan Academy, houve ganho significativo nesse quesito tanto nas turmas niveladas quanto nas desniveladas. Em 2015.2, por exemplo, a média de aprovação das turmas niveladas foi de $36,1 \%$; depois subiu para $69,6 \%$ em 2016.2 e diminuiu para 46,9\% em 2017.2, semestre no qual o nível de dificuldade da avaliação foi considerada adequada, conforme discussão anterior.

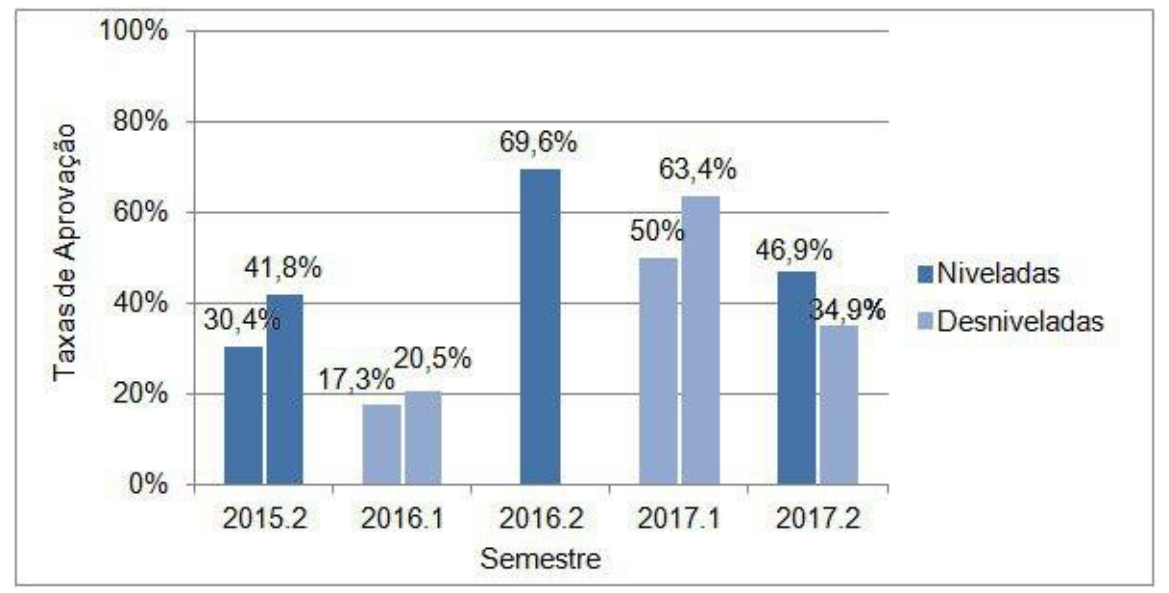

Gráfico 1. Taxas de aprovação das turmas desde 2015.2.

Ao analisarmos o impacto do uso da KA nas turmas de 2017.2, precisamos relembrar os motivos que nos fizeram separá-las em niveladas e desniveladas. O tempo dedicado à disciplina pelo aluno da turma desnivelada tende a ser menor em comparação ao da nivelada. Isso ocorre pelos mais variados motivos — tais como profissão, estágio, ou, até mesmo, desinteresse - 
que acarretam maiores índices de desistência. Na turma desnivelada, 48,5\% dos estudantes tiveram rendimento médio semestral abaixo dos 50\%. Se compararmos o desempenho da turma nivelada, essa porcentagem cai para $16,2 \%$ dos estudantes.

O Gráfico 2 traz as taxas de aprovação dos alunos em diferentes faixas de rendimento na KA, numa escala de 0 (zero) a 100\%. Na turma desnivelada, a primeira taxa de aprovação positiva, 38,5\%, aparece na faixa de aproveitamento de 50 a 80 pontos, e cresce nas demais faixas. Vale salientar que esse valor já é maior que a aprovação geral da turma, que foi de 34,9\%. $\mathrm{Na}$ turma nivelada, as duas primeiras faixas apresentam aprovação nula, mas somente 32,4\% dos alunos estão nessa faixa. Novamente, a primeira taxa de aprovação positiva, $50 \%$, já é maior que os 46,9\% gerais. Além disso, há um crescimento na taxa de aprovação na faixa de 90 a 100 pontos.

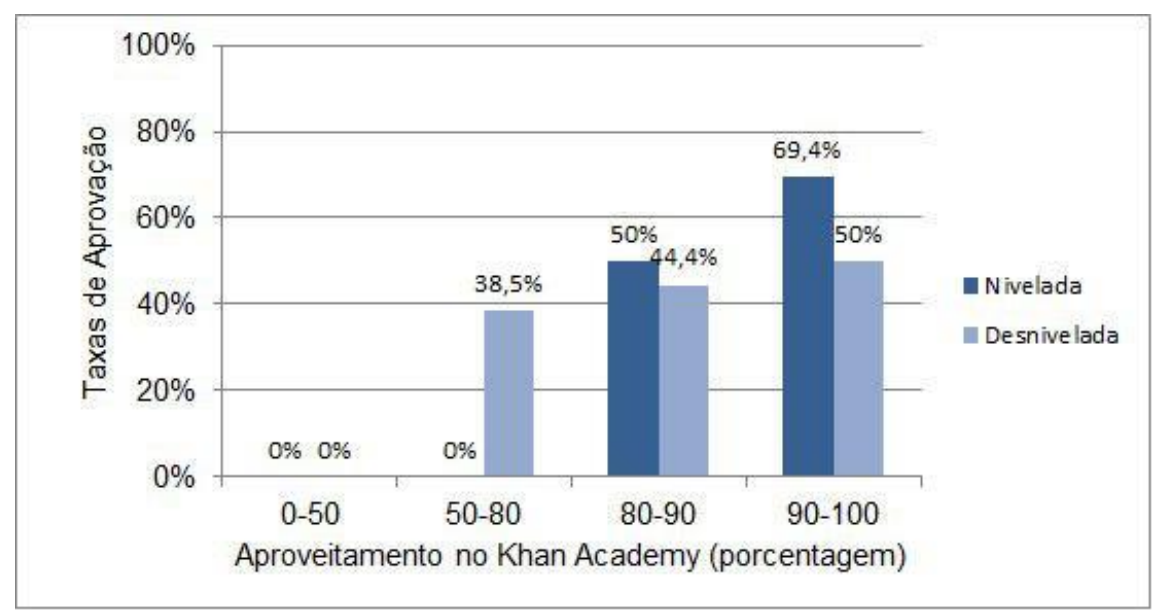

Gráfico 2. Taxas de aprovação das turmas de 2017.2.

Os Gráficos 1 e 2 consistem em uma análise totalmente quantitativa do impacto da prática empregada nas turmas de Cálculo I do BTI. Quando observado sob um viés qualitativo, o projeto também obteve resultados positivos, o que se evidencia pela comparação com semestres nos quais a integração da KA como prática de ensino ainda não havia sido implantada. Antes da integração, este proponente lecionou a disciplina cinco semestres, incluindo períodos em distintos departamentos. Após o início do uso dessa metodologia, a participação dos discentes em sala de aula aumentou devido ao estudo diário requerido para cumprir as atividades online. Essa análise torna notório o aproveitamento, por parte dos discentes, dos conteúdos ministrados a cada aula. Ademais, os estudantes vêm demonstrando apreço pela prática de ensino através de relatos. Muitos perguntam por que essa metodologia não é usada por outros professores. Mesmo os discentes repetentes que já tiveram contato com a proposta têm uma visão positiva sobre ela, relatando que a experiência foi benéfica apesar do insucesso na aprovação.

\section{Considerações Finais}

O uso da plataforma Khan Academy em sala de aula como descrito neste artigo foi de suma importância para melhorar o desempenho dos discentes na disciplina Cálculo I do Bacharelado em Tecnologia da Informação da UFRN. Apresentamos a necessidade de refinar o método avaliativo com o passar dos semestres, porém essa necessidade não invalida o uso da proposta. Pelo contrário, encoraja a buscar melhorias a cada semestre. 
VII Congresso Brasileiro de Informática na Educação (CBIE 2018)

Anais do XXIV Workshop de Informática na Escola (WIE 2018)

A partir do semestre 2018.1, a prática com a KA foi aplicada em turmas da disciplina Matemática Elementar, presente no primeiro semestre da grade curricular do curso. $\mathrm{O}$ método avaliativo foi composto por 2,0 pontos (em vez de 3,0) das atividades online e 8,0 pontos da prova escrita, que voltou a ser composta por questões fáceis, médias e difíceis. Uma pré-análise dessa distribuição trouxe a sensação de que, enfim, alcançamos um equilíbrio na avaliação que nos permita perceber os mais variados níveis de dificuldade também na prova escrita.

Uma dificuldade recorrente, que foi apenas citada, é a coleta braçal dos desempenhos dos estudantes. Com o intuito de otimizá-la, faremos um estudo mais apurado dos dados que a KA oferece para produzir uma ferramenta que obtenha os dados de forma automática.

\section{Refêrencias}

Bardy, L.R. et al (2007) "Os objetos de aprendizagem para pessoas com deficiência". In: Brasil. Objetos de aprendizagem: uma proposta de recurso pedagógico. Brasília: MEC/SEED.

Becker, F. (2012) Educação e construção do conhecimento. 2 ed. Porto Alegre: Penso, 200 p.

Castro-Filho et al. (2011) Objetos de Aprendizagem digitais como suporte para a construção e compreensão de gráficos. In: Anais da XIII CIEAEM. Recife: Edumatec/UFPE.

Medeiros Filho, D.A.; Moura, E.G.G. (2016) A Metodologia de Ensino da Khan Academy para a Área Tecnológica, Disponível em: 〈https://goo.gl/yiGTDb〉. Acesso em: 29 jun. 2018

Menegais, D.A.F.N.; Fagundes, L.C.; Sauer, L.Z. (2015) A Análise Do Impacto Da Integração Da Plataforma Khan Academy Na Prática Docente De Professores De Matemática. In: CINTED-UFRGS - Novas Tecnologias na Educação, V. 13 Nº 1, julho.

Morin, J. M. (2015) Mudando a educação com metodologias ativas. Coleção Mídias Contemporâneas. Convergências Midiáticas, Educação e Cidadania: aproximações jovens, v.2.

Papert, S. (2008) "A máquina das crianças: repensando a escola na Era da Informática”. ed. rev. Porto Alegre: Artmed.

Pavanelo, E.; Lima, R. (2017) Sala de Aula Invertida: a análise de uma experiência na disciplina de Cálculo I. In: Bolema, Rio Claro (SP), v. 31, n. 58, p. 739-759, ago.

QEDU (2018) Comparando o aprendizado: Rio Grande do Norte. Disponível em: http://www.qedu.org.br/estado/120-rio-grande-do-norte/compare.

Tavares, W. et al. (2014) Khan academy: uma abordagem da escola construtivista ou o uso de novas ferramentas na abordagem da escola tradicional da educação? RENOTE. Revista Novas Tecnologias na Educação, Porto Alegre, v.10, n. 1, p. 1-11, jul. 